\title{
A Theoretical Investigation on Thermal Entrance Region Heat Transfer in a Conduit Filled with Nanofluids
}

\author{
W. $\mathrm{Li}^{*}$ \\ Graduate School of Science and Technology, Shizuoka University, 3-5-1 Johoku, Naka-ku, Hamamatsu, 432-8561, Japan
}

\begin{abstract}
A theoretical analysis has been made to explore thermal entrance region heat transfer in nanofluids. The numerical model used in this paper is based on the Buongiorno model for convective heat transfer in nanofluids with modifications to fully account for the effects of nanoparticles volume fraction distributions on the continuity, momentum and energy equations. Numerical investigations have been conducted for developing laminar forced convection flows in a circular tube subject to a uniform wall heat flux. From the results, it has been concluded that, for certain cases of aluminawater nanofluids and titania-water nanofluids, in most entrance region, anomalous heat transfer enhancement (that exceeding the rate expected from the increase in thermal conductivity) is possible, whereas, close to the edge of the entrance, no anomalous heat transfer enhancement is observed.
\end{abstract}

Keywords: Nanofluids, laminar flow, entrance region, heat transfer enhancement, numerical simulation.

\section{INTRODUCTION}

Nanofluids refer to fluids containing dispersed nanosized solid particles as coined by Choi [1]. Because of their small size, nanoparticle suspensions are much more stable than millimeter and micrometer size colloids. This kind of feature allows nanofluids to enhance the thermal conductivity of the effective medium and heat transfer performance surpassing that of the base fluid without facing problems such as abrasion, clogging and high pressure loss. Therefore, the nanofluids are considered to be the next generation working fluids in modern heat transfer technologies $[2,3]$.

There are a great number of literatures on nanofluid convective heat transfer, most of them reported enhancement of convective heat transfer when using nanofluids [4-8]. However, when it comes to 'anomalous heat transfer enhancement, which refers to the heat transfer enhancement exceeding the rate expected from the increase in thermal conductivity, some nanofluids studies show inconsistency, reporting enhancement under some conditions and even deterioration under other conditions [9-11]. These have led to a controversial issue, namely, whether or not the anomalous convective heat transfer enhancement is possible in nanofluids. The controversy exists in both laminar and turbulent flows, especially for the entrance region. Rea et al. [12] presented experimental evidence that no abnormal enhancement occurs, including the entrance region. Ding et al. [13] also stressed that in some cases no enhancement takes place in the entrance region. However, based on certain statistical analysis methodology, Prabhat et al. [14] found that there is anomalous heat transfer enhancement in the entrance region in nanofluid laminar flows.

*Address correspondence to this author at the Graduate School of Science and Technology, Shizuoka University, 3-5-1 Johoku, Hamamatsu, 432-8561 Japan; Tel: +81-80-3683-3254; E-mail: f5245020@ipc.shizuoka.ac.jp
Some theoretical investigations on nanofluids convection also have been reported. A notable progress was made by Buongiorno [15] who carefully carried out a theoretical analysis so as to estimate relative magnitudes of the terms associated with all possible slip mechanisms, namely, inertia, Brownian diffusion, thermophoresis, diffusiophoresis, Magnus effect, fluid drainage and gravity, and concluded that only Brownian diffusion and thermophoresis are important in nanofluids. He derived a two-component fourequation nonhomogeneous equilibrium model for mass, momemtum and heat transfer in nanofluids, which has been used by many researchers including Tzou [16, 17], Hwang et al. [18], Nield and Kuznetsov [19].

In order to clarify the controversial issue, some numerical investigations have already been conducted to explore the convective heat transfer characteristic in nanofluids [20], in which only surface heat transfer coefficient was discussed without considering the variation of thermal conductivity. Moreover, it did not take into account of the effects of nanoparticles volume fraction distributions, which can affect the convective heat transfer via thermophysical properties.

In this paper, based on the Buongiorno model with modifications to fully account for the effects of nanoparticles volume fraction distributions on continuity, momentum and energy equations, numerical investigations have been conducted for developing laminar forced convection flow in a circular tube for certain cases as alumina-water nanofluids and titania-water nanofluids, so as to clarify the controversial issue on nanofluids anomalous convective heat transfer enhancement for the case of thermal entrance region.

\section{CONSERVATION EQUATIONS}

The theoretical description of the convective transport in nanofluids is given by Buongiorno [15], who assumed it to be an incompressible flow, no chemical reactions, negligible 


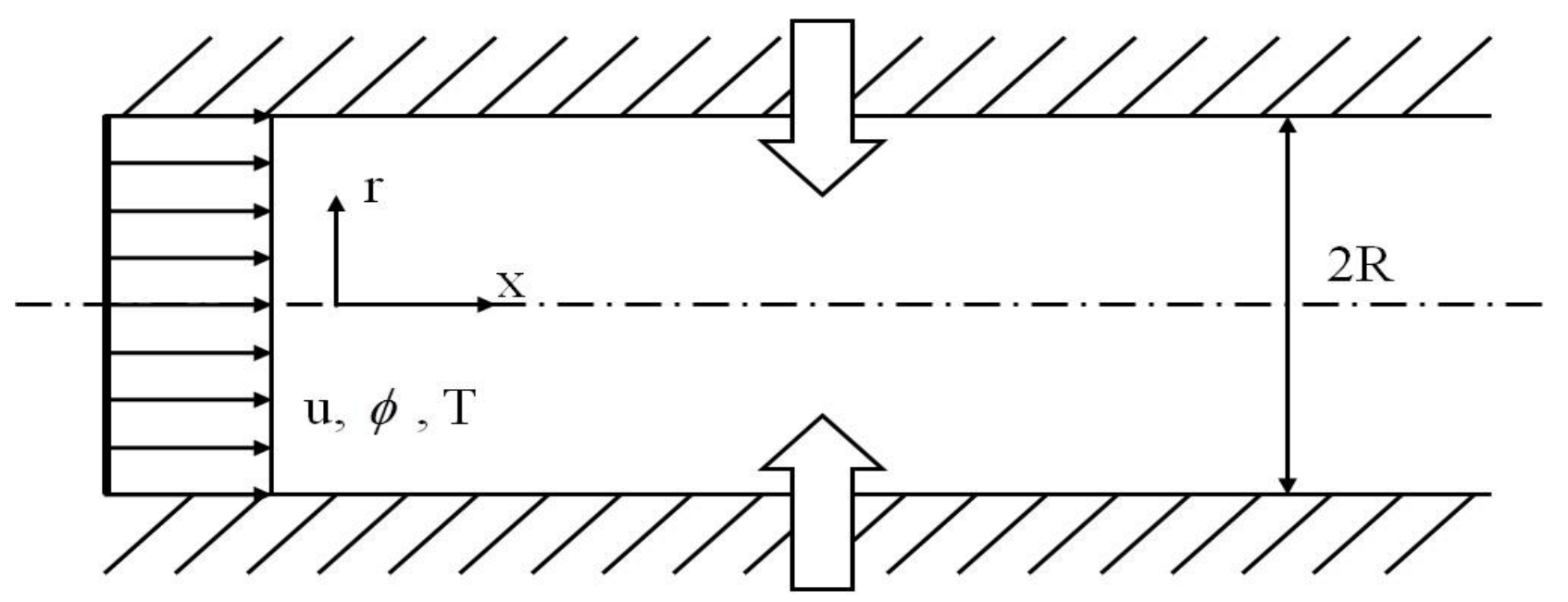

constant wall heat flux $\mathrm{q}_{\mathrm{w}}$

Fig. (1). Physical model.

external forces, dilute mixture, negligible viscous dissipation, negligible radiative heat transfer, and local thermal equilibrium between nanoparticles and base fluid. In this paper, the Buongiorno model may be modified to allow the nanofluid density $(\rho)$ variation in mass, momentum and energy conservations as

$$
\frac{\partial \rho u_{j}}{\partial x_{j}}=0
$$

$\frac{\partial \rho u_{i}}{\partial t}+\frac{\partial \rho u_{j} u_{i}}{\partial x_{j}}=-\frac{\partial p}{\partial x_{i}}+\frac{\partial}{\partial x_{j}} \mu\left(\frac{\partial u_{i}}{\partial x_{j}}+\frac{\partial u_{j}}{\partial x_{i}}\right)$

$\frac{\partial \rho c T}{\partial t}+\frac{\partial \rho c u_{j} T}{\partial x_{j}}=\frac{\partial}{\partial x_{j}} k \frac{\partial T}{\partial x_{j}}+\rho_{p} c_{p}\left(D_{B} \frac{\partial \phi}{\partial x_{j}}+\frac{D_{T}}{T} \frac{\partial T}{\partial x_{j}}\right) \frac{\partial T}{\partial x_{j}}$

$\frac{\partial \phi}{\partial t}+\frac{\partial u_{j} \phi}{\partial x_{j}}=\frac{\partial}{\partial x_{j}}\left(D_{B} \frac{\partial \phi}{\partial x_{j}}+\frac{D_{T}}{T} \frac{\partial T}{\partial x_{j}}\right)$

where $\rho, c, \mu$ and $k$ are the density, heat capacity, viscosity and thermal conductivity of the nanofluid, which depend on the nanoparticle volume fraction $\phi$ as

$$
\begin{aligned}
& \rho=\phi \rho_{p}+(1-\phi) \rho_{b f} \\
& c=\left(\phi \rho_{p} c_{p}+(1-\phi) \rho_{b f} c_{b f}\right) / \rho \\
& \mu=\mu_{b f}\left(1+a_{\mu} \phi+b_{\mu} \phi^{2}\right)=\left\{\begin{array}{c}
\mu_{b f}\left(1+39.11 \phi+533.9 \phi^{2}\right): \text { alumina } \\
\mu_{b f}\left(1+5.45 \phi+108.2 \phi^{2}\right): \text { titania }
\end{array}\right. \\
& k=k_{b f}\left(1+a_{k} \phi+b_{k} \phi^{2}\right)=\left\{\begin{array}{c}
k_{b f}(1+7.47 \phi): \text { alumina } \\
k_{b f}\left(1+2.92 \phi-11.99 \phi^{2}\right): \text { titania }
\end{array}\right.
\end{aligned}
$$

The results naturally depend on the correlations for thermophysical properties. The two sets of the correlations (5c) and (5d) represent high and low sensitivities with respect to the volume fraction of nanoparticles. Almost all correlations proposed so far, including Bianco et al. [21], stay within the range of these two sets of the correlations. range of these two sets of the correlations. Thus, the conclusions drawn from this study should generally apply to most correlations available today.

The Brownian and thermophoretic diffusion coefficients are given by

$D_{B}=\frac{k_{B O} T}{3 \pi \mu_{b f} d_{p}}$

and

$D_{T}=0.26 \frac{k_{b f}}{2 k_{b f}+k_{p}} \frac{\mu_{b f}}{\rho_{b f}} \phi$

respectively. The dimensionless parameter $N_{B T}$ which describes the ratio of Brownian and thermophoretic diffusivities is defined as follows:

$N_{B T} \equiv \frac{D_{B R} T_{w} \phi_{w} k_{w}}{D_{x_{v}} q_{w} R}=\frac{k_{B 0} T_{w}^{2} \rho_{b f} k_{w}}{3 \pi d_{p}\left(0.26 \frac{k_{b f}}{2 k_{b f}+k_{p}}\right) \mu_{b f}^{2} q_{w} R}$

The nanofluid thermophysical properties such as $\mu$ and $k$ are considered as given functions of $\phi$ as correlated by Buongiorno using the data of Pak and Cho [22], where the subscripts, $p$ and $b f$ refer to as nanoparticle and base fluid, respectively. Moreover, $k_{B O}$ is the Boltzmann constant and $d_{p}$ is the nanoparticle diameter, which can be anywhere of the order of 1 to $100 \mathrm{~nm}$. For typical cases of alumina and nanoparticles with $d_{p} \sim 10 \mathrm{~nm}$ and $\phi_{B} \sim 0.01$, the ratio of Brownian and thermophoretic diffusivities $N_{B T}$ can range from 0.1 to 10 . Buongiorno proved that the heat transfer associated with the nanoparticle dispersion (i.e. nanoparticle diffusion flux), namely, the second term on the right hand side of Eq. (3), is always negligible as compared with convection and conduction.

\section{NUMERICAL METHOD}

Fig. (1) shows the physical model under consideration in this study. It consists of a circular tube with the ratio of length and diameter $\mathrm{L} / \mathrm{D}=100$, and the tube wall is subject to 


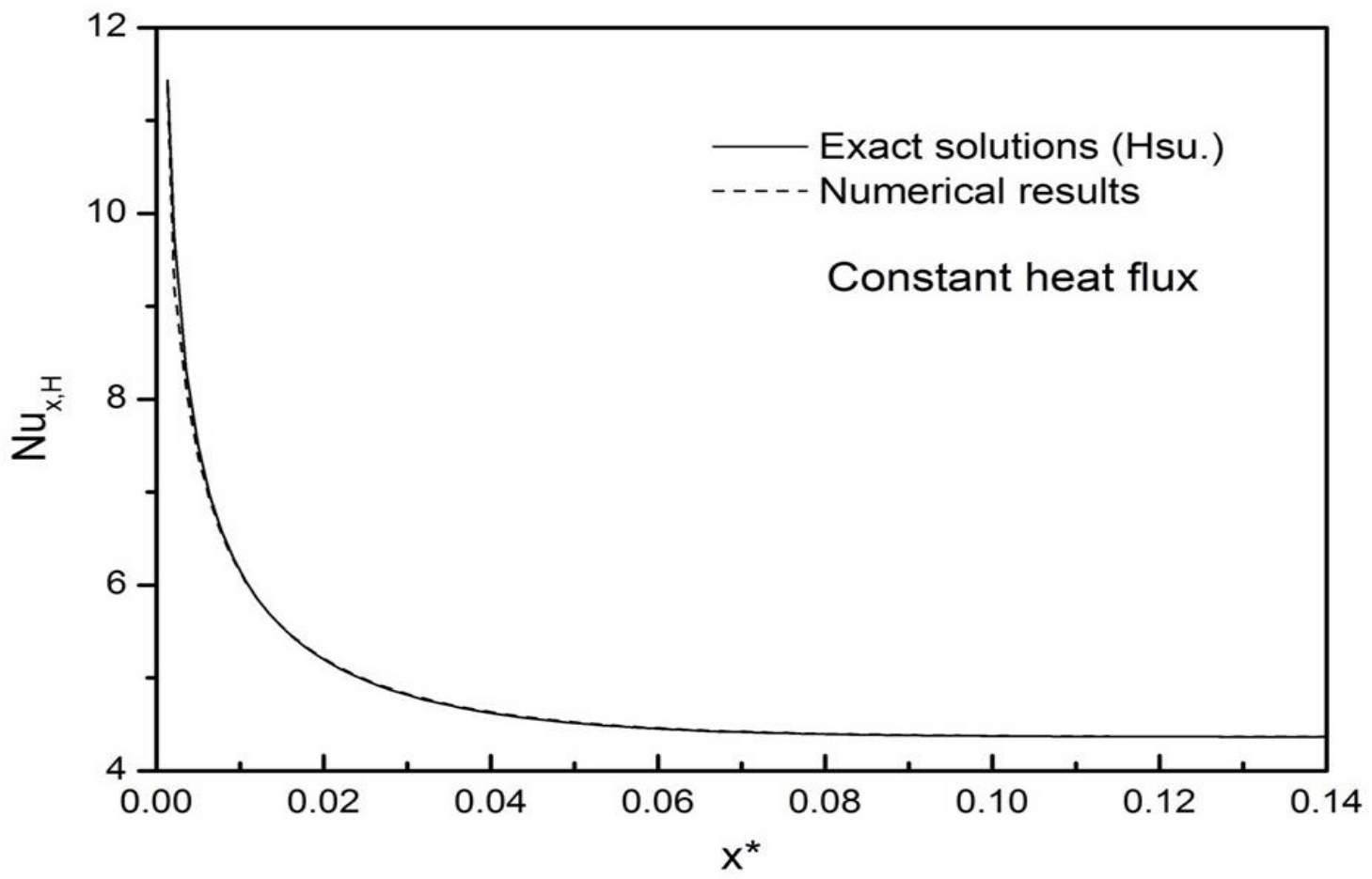

Fig. (2). Local Nusselt number for thermally developing flow of pure fluid.

a uniform heat flux $q_{w}$. The tube has an appropriate length to ensure the computational domain stays within the entrance region. The fluid enters with uniform nanoparticle distribution, temperature and axial velocity profiles at the inlet section. Also, the nanoparticle volume fraction, flow and thermal field are assumed to be symmetrical so that one meridian plane of the tube is considered in the numerical experiments.

The foregoing governing equations (1)-(4) are solved by integrating them over finite control volumes, converting the governing equations to a set of discretized equations. The numerical program was established to these algebraic discretized equations. As proposed by Patankar and Spalding [23], SIMPLE algorithm was adopted to correct the pressure and velocity fields. Further details on this numerical procedure can be found in Patankar [24] and Nakayama [25].

In order to evaluate the heat transfer enhancement of nanofluid, the local Nusselt number along the axial distance of the tube should be introduced at first. The local Nusselt number based on the diameter $D_{h}=2 R$ and the nanofluid bulk thermal conductivity $k_{B}=k\left(\phi_{B}\right)$ are given by

$$
N u_{B}(x) \equiv \frac{h(x) D_{h}}{k_{B}}=\frac{q_{w}(2 R)}{\left(T_{w}(x)-T_{B}(x)\right) k_{B}}
$$

where

$$
T_{B} \equiv \frac{\langle\rho c u T\rangle}{\langle\rho c u\rangle}
$$

denotes the bulk mean temperature, and

$$
\phi_{B} \equiv \frac{\langle u \phi\rangle}{\langle u\rangle}
$$

denotes the bulk mean particle volume fraction, and

$$
\langle\varphi\rangle \equiv \frac{1}{A} \int_{A} \varphi d A=\frac{1}{\pi R^{2}} \int_{0}^{R} 2 \pi r \varphi d r
$$

denotes average value over the cross-section.

\section{NUMERICAL VALIDATION}

The validation of grid dependency was conducted to ensure the accuracy of the numerical results, and found that a grid system $101 \times 51$ is fine enough for each of the cases considered. Then, Graetz problem [26] for thermally developing flow of pure fluid was used to verify the numerical program. Fig. (2) presented the comparison of exact solutions and numerical results for thermally developing flow of pure fluid, which agrees well with each other.

Moreover, the numerical program was used to calculate the local Nusselt number $N u_{B}$ of fully developed nanofluids flow and compared with exact solutions which had already been obtained by Yang et al. [27]. Comparison of exact solutions and numerical results for fully developed flow of titania-water nanofluids and alumina-water nanofluids are presented in Table $\mathbf{1}$ and $\mathbf{2}$, respectively. The results shows that, for different bulk mean particle volume fractions $\phi_{B}$, the ratio of Brownian and thermophoretic diffusivities $N_{B T}$ and different type of nanofluids, the numnerical results and exact solutions are almost identical under consideration of numerical error. The foregoing comparison for validation reveals that the numerical program we adopted is quite accurate.

\section{RESULTS AND DISCUSSION}

For nanofluids with different bulk mean particle volume fractions $\left(\phi_{B}=0.02,0.06,0.1\right)$ and pure fluid $\left(\phi_{B}=0\right)$, the local Nusselt number is calculated under the same mass flow 
Table 1. Local Nusselt Number for Fully Developed Flow of Titania-Water Nanofluid

\begin{tabular}{|c|c|c|c|c|c|c|}
\hline \multirow{2}{*}{$\begin{array}{c}\varphi_{\mathrm{B}} \\
\mathbf{N}_{\mathrm{BT}}\end{array}$} & \multicolumn{2}{|c|}{0.02} & \multicolumn{2}{|c|}{0.06} & \multicolumn{2}{|c|}{0.10} \\
\hline & Exa & Num & Exa & $\mathbf{N}_{\mathrm{BT}}$ & Exa & Num \\
\hline 0.1 & 4.277 & 4.278 & 4.234 & 4.235 & 4.200 & 4.201 \\
\hline 1 & 4.372 & 4.371 & 4.458 & 4.456 & 4.575 & 4.573 \\
\hline 10 & 4.365 & 4.366 & 4.377 & 4.378 & 4.392 & 4.393 \\
\hline
\end{tabular}

Table 2. Local Nusselt Number for Fully Developed Flow of Alumina-Water Nanofluid

\begin{tabular}{|c|c|c|c|c|c|c|}
\hline$\frac{\varphi_{\mathrm{B}}}{\mathbf{N}_{\mathrm{BT}}}$ & \multicolumn{2}{|c|}{0.02} & \multicolumn{2}{|c|}{0.06} & \multicolumn{2}{|c|}{0.10} \\
\hline 0.1 & 4.211 & 4.212 & 4.019 & 4.019 & 3.941 & 3.934 \\
\hline 1 & 4.418 & 4.415 & 4.432 & 4.423 & 4.414 & 4.401 \\
\hline 10 & 4.371 & 4.372 & 4.374 & 4.373 & 4.372 & 4.371 \\
\hline
\end{tabular}

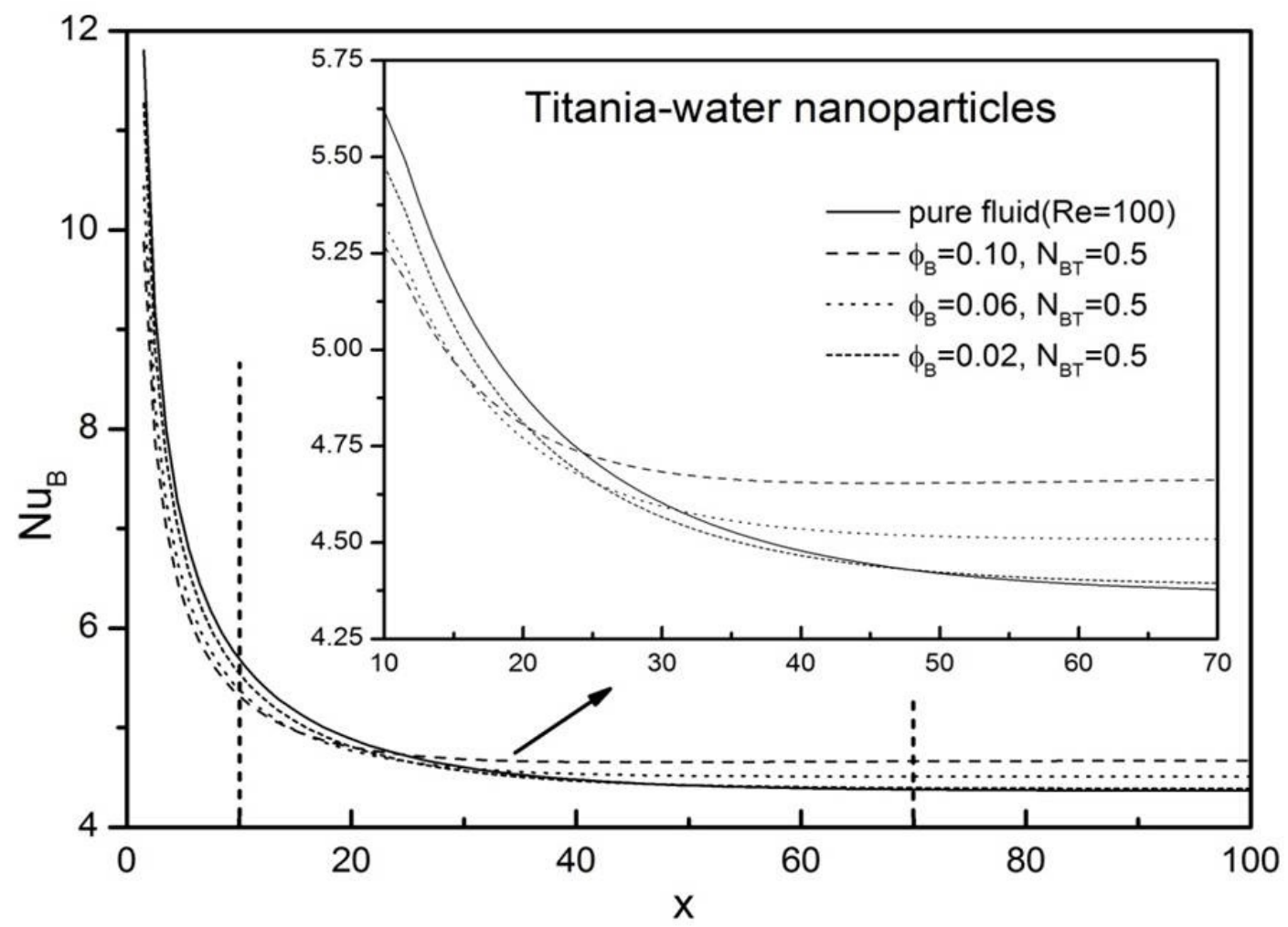

Fig. (3). Local Nusselt number for developing flow of titania-water nanofluid.

rate. The Reynold number of pure fluid is set to 100. As mentioned above, the ratio of Brownian to thermophoretic diffusivities $N_{B T}$ can range from 0.1 to 10 . Here, we present typical results obtained under $N_{B T}=0.5$.

Local Nusselt number for titania-water nanofluids in a circular tube is presented in Fig. (3). It shows that, for fixed $N_{B T}=0.5$, in the initial part of entrance region, $N u_{B}$ stays below the value of the pure fluid, which means there is no anomalous heat transfer enhancement, but in most of entrance region, $N u_{B}$ stays above the value of pure fluid indicating that anomalous heat transfer enhancement exists. It also can be seen that more extensitve heat transfer anomaly is possible at a higher $\phi_{B}$ (e.g. $7 \%$ increase when $\phi_{B}=0.1$ ).

Fig. (4) presents the results for alumina-water nanofluids. As in the previous case of titania-water nanofluids, there is anomalous heat transfer enhancement in the major part of entrance region, but no anomalous heat transfer enhancement in the leading edge of entrance region. However, for alumina-water nanofluids, the anomaly level of heat transfer enhancement does not increase monotonously with $\phi_{B}$. Form Fig. (4), we can see that when $\phi_{B}=0.06$, the anomalous heat transfer enhancement is the highest among the three cases, 


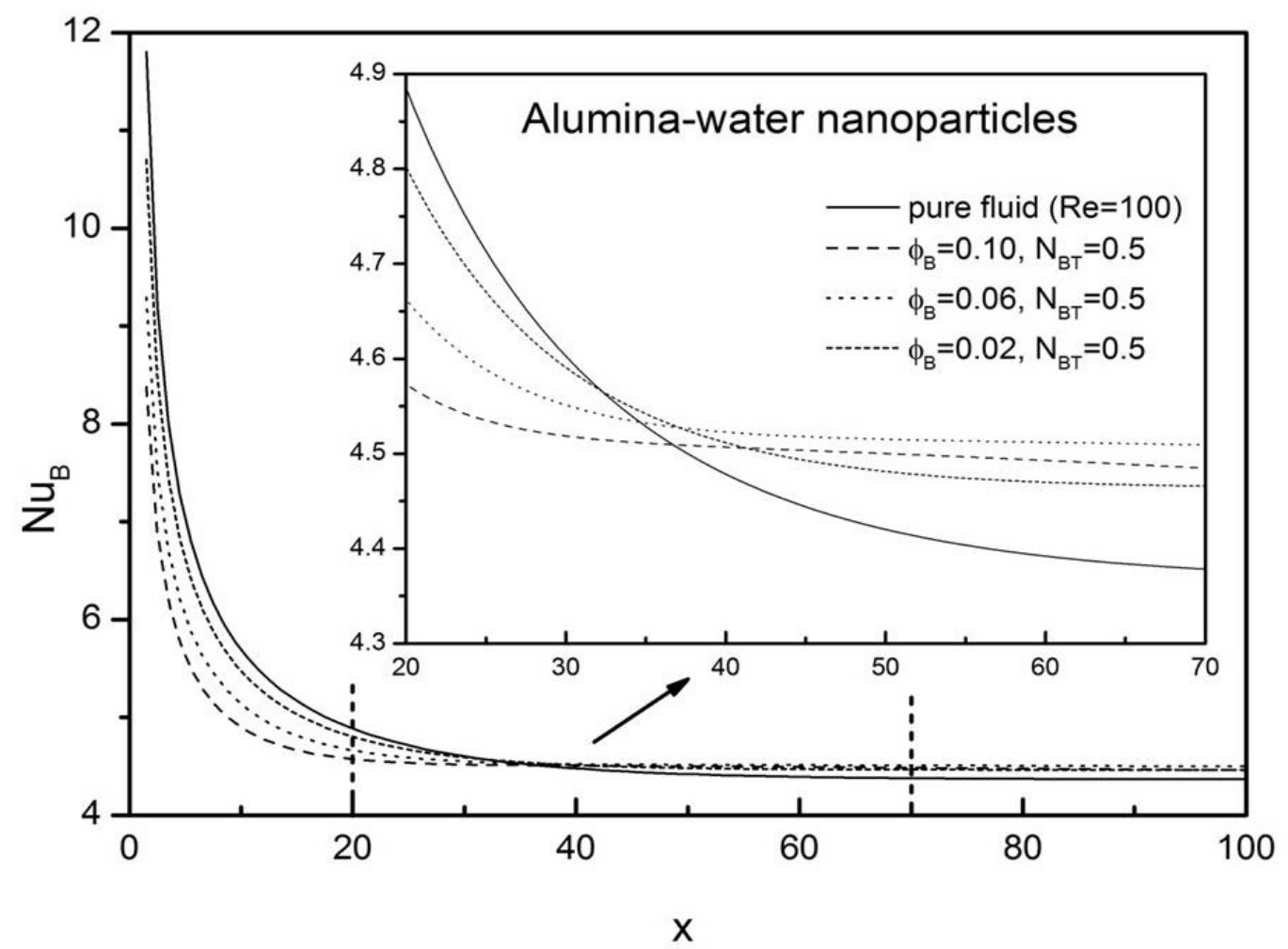

Fig. (4). Local Nusselt number for developing flow of alumina-water nanofluid.

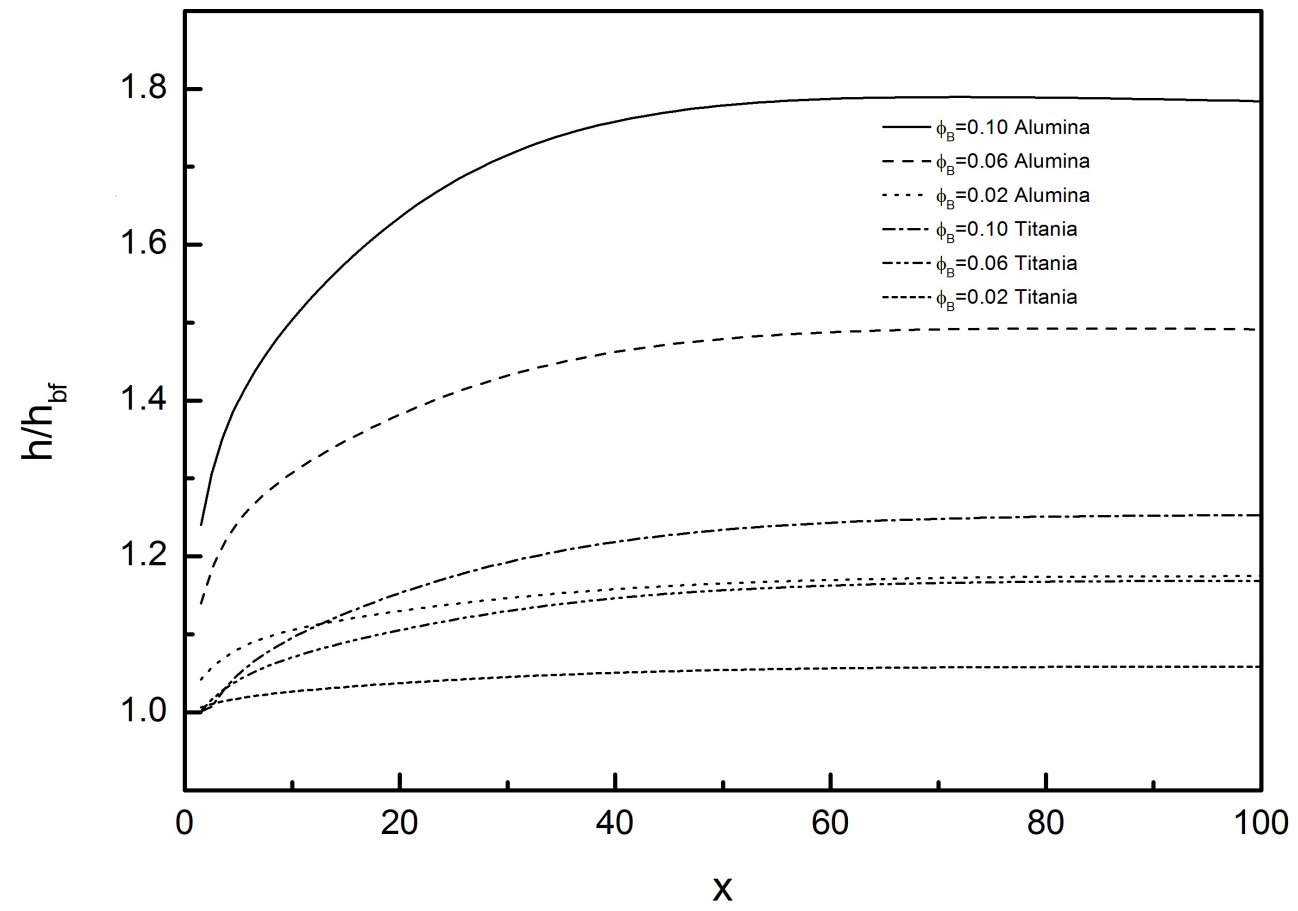

Fig. (5). Comparison of the heat transfer coefficients of nanofluids.

namely, $\phi_{B}=0.02,0.06$ and 0.10 . This indicates that the level of the anomaly is very sensitive to the properties of the nanofluids.

It should be mentioned that, according to Eq. (8), the heat transfer enhancement may be evaluated as the ratio of heat transfer coefficient of nanofluids and base fluid, $\frac{h}{h_{b f}}=\frac{N u_{B}}{N u_{b f}}\left(\frac{k_{B}}{k_{b f}}\right)=\frac{N u_{B}}{N u_{b f}}\left(1+a_{k} \phi_{B}+b_{k} \phi_{B}^{2}\right)$

Fig. (5) clearly shows that other than the complex manner of anomalous heat transfer enhancement, the heat transfer enhancement of titania-water nanofluids and alu- 
mina-water nanofluids is remarkable. Furthermore, the heat transfer enhancemet of alumina-water nanofluids is higher than that of titania-water nanofluids, simply due to the difference in $k_{B}$.

\section{CONCLUSIONS}

Numerical investigations have been conducted to explore the thermal entrance region heat transfer in nanofluids. The effects of nanoparticle volume fraction distributions on the continuity, momentum and energy equations have been fully considered based on the Buongiorno model. For both of alumina-water nanofluids and titania-water nanofluids, anomalous heat transfer enhancement have been captured in the major part of the entrance region, However, no anomalous heat transfer enhancement is found close to the edge of the entrance region. Moreover, the anomaly level of heat transfer enhancement increases as increasing the bulk mean particle volume fraction for titania-water nanofluids, whereas this is not the case for alumina-water nanofluids. As observed in the present analysis, the entrance region heat transfer enhancement of nanofluids is quite complex. A further study is required to elucidate more details of the heat transfer anomaly associated with nanofluids.

\section{NOMENCLATURE}

\begin{tabular}{|c|c|c|}
\hline$A$ & $=$ & cross-sectional area $\left[\mathrm{m}^{2}\right]$ \\
\hline$C$ & $=$ & specific heat of nanofluid $[\mathrm{J} / \mathrm{kgK}]$ \\
\hline$d_{p}$ & $=$ & nanoparticle diameter $[\mathrm{m}]$ \\
\hline$D_{B}$ & $=$ & $k_{B O} T / 3 \pi \mu_{b f} d_{p}$, Brownian diffusivity [m2/s] \\
\hline $\mathrm{D}_{\mathrm{T}}$ & $=$ & $\begin{array}{l}0.26 \frac{\mathrm{k}_{\mathrm{bf}}}{2 \mathrm{k}_{\mathrm{bf}}+\mathrm{k}_{\mathrm{p}}} \frac{\mu_{\mathrm{bf}}}{\rho_{\mathrm{bf}}} \mathrm{j} \text {, thermophoretic diffusivity } \\
{[\mathrm{m} 2 / \mathrm{s}]}\end{array}$ \\
\hline$D_{h}$ & $=$ & hydraulic diameter [m] \\
\hline$h$ & $=$ & heat transfer coefficient $\left[\mathrm{W} / \mathrm{K} \mathrm{m}^{2}\right]$ \\
\hline$k$ & $=$ & thermal conductivity $[\mathrm{W} / \mathrm{m} \mathrm{K}]$ \\
\hline$k_{B O}$ & $=$ & Boltzmann constant $[\mathrm{J} / \mathrm{K}]$ \\
\hline$N_{B T}$ & $=$ & $\begin{array}{l}\text { ratio of Brownian and thermophoretic dif- } \\
\text { fusivities [-] }\end{array}$ \\
\hline$N u_{B}$ & $=$ & $\begin{array}{l}\mathrm{hD}_{\mathrm{h}} / \mathrm{k}_{\mathrm{B}} \text {, Nusselt number based on the bulk } \\
\text { mean thermal conductivity [-] }\end{array}$ \\
\hline$p$ & $=$ & pressure $[\mathrm{Pa}]$ \\
\hline$q_{w}$ & $=$ & wall heat flux $\left[\mathrm{W} / \mathrm{m}^{2}\right]$ \\
\hline$r$ & $=$ & radial coordinate $[\mathrm{m}]$ \\
\hline$R$ & $=$ & tube radius $[\mathrm{m}]$ \\
\hline$T$ & $=$ & temperature $[\mathrm{K}]$ \\
\hline$u$ & $=$ & axial velocity $[\mathrm{m} / \mathrm{s}]$ \\
\hline$u_{i}$ & $=$ & velocity vector $[\mathrm{m} / \mathrm{s}]$ \\
\hline$x$ & $=$ & axial coordinate $[\mathrm{m}]$ \\
\hline$y$ & $=$ & vertical coordinate $[\mathrm{m}]$ \\
\hline
\end{tabular}

\section{GREEK SYMBOLS}

$\begin{array}{lll}\mu & = & \text { viscosity of nanofluid }[\mathrm{Pa} \mathrm{s}] \\ \rho & = & \text { density of nanofluid }[\mathrm{kg} / \mathrm{m} 3] \\ \phi & = & \text { volume fraction of nanoparticles [-] }\end{array}$

\section{SPECIAL SYMBOLS}

$\langle\varphi\rangle \quad=\quad$ average over the cross-section

\section{SUBSCRIPTS AND SUPERSCRIPTS}

$\begin{array}{lll}B & = & \text { bulk mean } \\ b f & = & \text { base fluid } \\ p & = & \text { nanoparticle } \\ w & = & \text { wall }\end{array}$

\section{CONFLICT OF INTEREST}

The authors confirm that this article content has no conflicts of interest.

\section{ACKNOWLEDGEMENT}

Declared none.

\section{REFERENCES}

[1] S.U.S. Choi, "Enhancing Thermal Conductivity of Fluids with Nanoparticle," In: Developments and Applications of NonNewtonian Flows, D. A. Soginer, and H. P. Wang, Eds., ASME: USA, FED-vol. 231/MD-vol. 66, pp. 99-105, 1995.

[2] O. Mahian, A. Kianifar, S.A. Kalogirou, I. Pop, S. Wongwises, “A review of the applications of nanofluids in solar energy", Int. J. Heat Mass Transf., vol. 57, pp. 582-594, 2013.

[3] K. F. V. Wong, O.D. Leon, "Applications of nanofluids: current and future", Adv. Mech. Eng., 519659, 2010.

[4] S. Kaka, A. Pramuanjaroenkij, "Review of convective heat transfer enhancement with nanofluids", Int. J. Heat Mass Transf, vol. 52, no. 13-14, pp. 3187-3196, 2009.

[5] Y. Xuan, W. Roetzel, "Conceptions for Heat Transfer Correlations of Nanofluids”, Int. J. Heat Mass Transf, vol. 43, pp. 3701-3707, 2000.

[6] S. Lee, S. U. S. Choi, "Application of Metallic Nanoparticle Suspensions in Advanced Cooling Systems", Int. Mechanical Engineering Congress and Exhibition, Atlanta, U.S.A., pp. 1-12, 1996.

[7] S. P. Jang, S. U. S. Choi, "Cooling Performance of a Microchannel Heat Sink with Nanofluids", Appl. Therm. Eng., vol. 26, pp. 24572463, 2006.

[8] S. Z. Heris, M. N. Esfahany, S. G.. Etemad, "Experimental Investigation of Convective Heat Transfer of $\mathrm{Al}_{2} \mathrm{O}_{3}$ /Water Nanofluid in a Circular Tube", Int. J. Heat Fluid Flow, vol. 28, pp. 203-210, 2007.

[9] B. C. Pak, Y. Cho, "Hydrodynamic and Heat Transfer Study of Dispersed Fluids with Submicron Metallic Oxide Particles", Exp. Heat Transf., vol. 11, pp. 151-170, 1998.

[10] R. Chien, J. Chuang, "Experimental Micro-channel Heat Sink Performance Studies Using Nanofluids", Int. J. Therm. Sci., vol. 46, pp. 57-66, 2007.

[11] J. Lee, I. Mudawar, "Assesment of the Effectiveness of Nanofluids for Single Phase and Two-Phase Heat Transfer in Microchannels", Int. J. Heat Mass Transf., vol. 50, pp. 452-463, 2007.

[12] U. Rea, T. McKrell, L.W. Hu, J. Buongiorno, "Laminar convective heat transfer and viscous pressure loss of alumina-water and zirconia-water nanofluids", Int. J. Heat Mass Transf., vol. 52, pp. 2042-2048, 2009.

[13] Y. Ding, H. Chen, L. Wang, C.Y. Yang, Y. He, W. Yang, W. P. Lee, L. Zhang, R. Huo, "Heat transfer intensification using nanofluids", KONA, pp. 23-38, no. 25, 2007. 
[14] N. Prabhat, J. Buongiorno, L.W. Hu, "Convective heat transfer enhancement in nanofluids: Real anomaly or analysis artifact?" Proc. the ASME/JSME 2011 8th Thermal Engineering Joint Conference, AJTEC2011, Honolulu, Hawaii, USA, pp. 1-10, March 13-17, 2011.

[15] J. Buongiorno, "Convective transport in nanofluids", J. Heat Transf., vol. 128, pp. 240-250, 2006.

[16] D. Y. Tzou, "Instability of nanofluids in natural convection", $J$. Heat Transf., vol. 128, pp. 240-250, 2008.

[17] D. Y. Tzou, "Thermal instability of nanofluids in natural convection”, Int. J. Heat Mass Transf., vol. 51, pp. 2967-2979, 2008.

[18] K. S. Hwang, S. P. Jang, S. U. S. Choi, "Flow and convective heat transfer characteristics of water-based $\mathrm{Al} 2 \mathrm{O} 3$ nanofluids in fully developed laminar flow regime", Int. J. Heat Mass Transf., vol. 52, pp. 193-199, 2009.

[19] D. A. Nield, A. V. Kuznetsov, "Thermal instability in a porous medium layer saturated by a nanofluid", Int. J. Heat Mass Transf., vol. 52, pp. 5796-5801, 2009.

[20] S. Tahir, M. Mital, "Numerical investigation of laminar nanofluid developing flow and heat transfer in a circular channel", Appl Therm. Eng., vol. 39, pp. 8-14, 2012.
[21] V. Bianco, F. Chiacchio, O. Manca, S. Nardini, "Numerical investigation of nanofluids forced convection in circular tubes", Appl Therm Eng., vol. 29, no. 17-18, pp. 3632-3642, 2009.

[22] B. C. Pak, Y. Cho, "Hydrodynamic and heat transfer study of dispersed fluids with submicron metallic oxide particles", Exp. Heat Transf., vol. 11, pp. 151-170, 1998.

[23] S. V. Patankar, D.B. Spalding, "A calculation procedure for heat mass and momentum transfer in three-dimensional parabolic flows", Int. J. Heat Mass Transf., vol. 15, pp. 1787-1806, 1972.

[24] S.V. Patankar, Numerical heat transfer and fluid flow, Hemisphere Publishing Cooperation: Washington D.C., 1980.

[25] A. Nakayama, PC-aided numerical heat transfer and convective flow, CRC Press: Boca Raton, 1995.

[26] R.K. Shah, A.L. London, Laminar Flow ForcedConvection in Ducts, Supplement to Advances in Heat Trans., Academic Press, New York, pp.78-79, 196-203, 1978.

[27] C. Yang, W. Li, Y. Sano, M. Mochizuki, A. Nakayama, "On the anomalous convective heat transfer enhancement in nanofluids: A theoretical answer to the nanofluids controversy", J. Heat Transf., vol. 135, no. 054504-1, 2013.

(C) W. Li; Licensee Bentham Open.

This is an open access article licensed under the terms of the Creative Commons Attribution Non-Commercial License (http://creativecommons.org/licenses/by-nc/3.0/) which permits unrestricted, non-commercial use, distribution and reproduction in any medium, provided the work is properly cited. 